\title{
Étude sémantique des unités censé / réputé / supposé + infinitif : les limites de la synonymie
}

\section{Corinne Féron et Danielle Coltier}

\section{(2) OpenEdition \\ 1 Journals}

\section{Édition électronique}

URL : https://journals.openedition.org/pratiques/1329

DOI : 10.4000/pratiques.1329

ISSN : 2425-2042

Éditeur

Centre de recherche sur les médiations (CREM)

\section{Édition imprimée}

Date de publication : 15 juin 2009

Pagination : 150-164

\section{Référence électronique}

Corinne Féron et Danielle Coltier, «Étude sémantique des unités censé / réputé / supposé + infinitif : les limites de la synonymie », Pratiques [En ligne], 141-142 | 2009, mis en ligne le 20 juin 2014, consulté le 12 mars 2023. URL : http://journals.openedition.org/pratiques/1329 ; DOI : https://doi.org/10.4000/ pratiques. 1329 


\section{Etude sémantique des unités censé / réputé / supposé + infinitif : les limites de la synonymie}

\section{Corinne Féron, Danielle Coltier}

Université du Mans

\& CELTED, Université Paul-Verlaine, Metz

Notre point de départ ${ }^{(1)}$ réside dans ce constat ${ }^{(2)}$ : dans tous les dictionnaires de langue que nous avons consultés ${ }^{(3)}$, censé est défini par réputé ; s'y ajoute, presque toujours, supposé(4).

Nous nous proposons de voir en quoi ces unités peuvent être rapprochées - et en quoi elles diffèrent; notre travail est centré sur censé : il s'agit donc de décrire le sens en énoncé de cette unité, par comparaison avec réputé et supposé. Nos exemples sont puisés principalement dans les textes de Frantext intégral ${ }^{(5)}$ postérieurs à 1900.

Nous ne prenons en compte que la construction commune aux trois unités $(\mathrm{du}$ moins en français contemporain standard) : X (être) unité INF ; réputé et supposé ne seront donc étudiés que partiellement : une étude complète de ces unités exigerait la prise en compte des autres constructions (supposé / réputé + attribut, réputé pour inf, à quoi il faudrait ajouter les emplois sans complément : un vin réputé, une existence supposée).

(1) Nous remercions de leur aide les participants de l'atelier de linguistique de l'Université de Metz.

(2) Nous suivons les « rectifications de l'orthographe» proposées en 1990.

(3) Dictionnaire généraux du français contemporain ou incluant le français contemporain : GLLF, Lexis, GR, NPRE, TLFi, Ac. 9. Dans certains de ces dictionnaires, censédonne lieu à une seconde définition: GLLF (après la marque d'usage «classique ») : "Rangé dans une catégorie, un groupe »; TLFi: «syn de censément », emploi signalé comme «populaire».

(4) Lorsque supposé ne figure pas dans la définition de censé, il est donné comme synonyme (dans le GLLF). A l'inverse, censé n'apparait pas dans les articles consacrés à supposé et à réputé (dans les dictionnaires qui définissent ces unités), sauf dans le GR qui signale 1'usage de être réputé + inf. en droit et donne comme définition « être censé (avoir fait telle chose, être dans telle situation, etc.) ».

(5) http://www.frantext.fr/ 


\section{Généralités}

\subsection{Points communs aux trois unités}

Du point de vue syntaxique, ces trois unités partagent donc la construction $X$ (être) unité INF (où INF désigne l'infinitif et ses compléments éventuels); l'élément $X$ est le « contrôleur » de l'infinitif ; contrôleur et infinitif construisent un contenu propositionnel (on l'appellera $p$ ).

Du point de vue sémantique, on note que dans leur usage courant (hors vocabulaire «spécialisé » ${ }^{(6)}$ ), ces unités sont neutres au regard de la question de la prise en charge de la vérité du contenu propositionnel : les suites $X$ (être) réputé/censé/ supposé INF ne laissent pas prévoir si $p$ est ou non pris en charge par le locuteur : il peut l'être, ne pas l'être ou rester indéterminé de ce point de vue.

\subsection{Différences entre les unités}

\subsubsection{Différences syntaxiques et catégorielles}

a) L'infinitif est la seule construction possible pour $c e n s e^{(7)}$ alors que pour réputé et supposé, $X$ (être) unité INF n'est qu'une construction (relativement peu fréquente) à côté de $X$ (être) unité + attribut, cet attribut étant un adjectif, un participe passé, voire un groupe nominal.

b) Supposé et réputé peuvent être analysés comme des participes passés, respectivement des verbes supposer et réputer ${ }^{(8)}$, même si ces participes peuvent ne plus correspondre, sémantiquement, aux verbes. Censé en revanche ne peut être mis en relation avec un infinitif; de ce fait, la catégorisation de cette unité pose problème :

- tous les dictionnaires consultés considèrent censé comme un adjectif; or, la construction de censén'est pas une construction adjectivale : quand l'adjectif admet comme complément un infinitif, celui-ci est prépositionnel ; Gross (1975 : 164), qui catégorise censé - mais aussi réputé -comme adjectif, note que ce sont les seuls adjectifs à admettre un complément direct ;

- une autre analyse consiste à voir dans être censé une construction passive (Wilmet 2003 : 500, § 581, Le Goffic 1993 : 202-203). Le Bon Usage (2007 : $986, \S 772, \mathrm{~g}$ ) note que dans le cas où le participe subsiste seul (comme c'est le cas de marri, par exemple, de l'ancien verbe marrir), «il est préférable de considérer qu'il s'agit d'adjectifs » et ajoute que «être censé est pourtant resté proche de la valeur verbale » : il est, de ce fait, cité parmi les verbes qui, au passif, se construisent avec un infinitif (dont réputer et supposer), « avec la différence qu'il est figé au passif $»(\$ 772, \mathrm{f} 2)^{(9)}$.

\subsubsection{Différences sémantiques (hors vocabulaire spécialisé)}

a) La possibilité de substitution de réputé à supposé ou censé n'est pas régulière : impossible entre (1) et (2), elle est possible dans (3) :

(6) i.e. hors textes juridiques et théoriques.

(7) Censé INF est la seule construction courante en français standard. Censé+ adjectif ou GN, présenté par le $T L F i$ et le $G R$ comme un cas d'ellipse de être, est attesté dans le corpus Frantext.

(8) Rare en français contemporain : $c f$. GLLF, qui signale que réputer " ne s'emploie plus qu'au passif suivi d'un attribut ou d'un infinitif ».

(9) Nous nous rallions, quoique de façon encore très intuitive, à cette analyse. 
(1) Il est censé / supposé venir ce soir.

(2) *Il est réputé venir ce soir.

(3) Il est censé / supposé / réputé venir souvent / le soir.

L'impossibilité semble due au fait qu'avec réputé, le procès dénoté par l'infinitif doit avoir connu au moins une occurrence empirique (une actualisation) antérieure au moment où (être) réputé est valide ; censé et supposé ne présentent pas cette contrainte sémantique.

b) Quand elle est possible, la commutation entraine des changements sémantiques, nets lorsqu'on remplace réputé par supposé ou censé (ou inversement), moins évidents lorsque la commutation concerne censé et supposé. Nous ferons les hypothèses suivantes concernant les différences sémantiques entre les trois unités:

- réputé a un trait médiatif ${ }^{(10)}$ inhérent à sa signification : réputé indique que $p$ est acquis par emprunt; un tel trait n'est inhérent ni à censé, ni à supposé; ces unités peuvent l'acquérir contextuellement;

- censé et supposé fournissent d'abord une indication sur le mode d'existence du contenu propositionnel $p$ correspondant à $X I N F$ : ce que dénote INF est présenté comme virtuel, idéel.

\section{CENSÉ}

Nous proposons de distinguer deux significations de censé. Dans la première (censé1), censé signifie une inférence ${ }^{(11)}$ du locuteur; dans l'autre (censé2), censé signifie un jugement ${ }^{(12)}$.

\subsection{Censé1}

\subsubsection{Observations générales sur censé1}

L’énoncé (4) :

(4) Jules est censé étudier.

admet les enchainements suivants :

(5) Jules est censé étudier. Mais est-ce le cas (qu'il étudie)?

(6) Jules est censé étudier. Et il étudie. / Mais il n'étudie pas.

Ces enchaînements ne conviennent pas à (7) :

(10) Nous préférons médiatif à évidentiel et nous adoptons cette définition (où l'on remplacera évidentialité par médiativité) : «Dans la conception large, l'évidentialité englobe la notion de modalité comme expression de l'attitude épistémique du locuteur. Dans la conception étroite, l'évidentialité est le pendant et le complément épistémique de la modalité. [...] on ne peut que gagner en clarté si l'on oppose, plutôt qu'on n'intègre, les concepts de modalité et d'évidentialité et qu'on réserve donc le terme de modalité à l'expression de l'attitude du locuteur et celui d'évidentialité à l'expression du mode de création et / ou de récolte de l'information, quitte à utiliser éventuellement un autre terme - par exemple celui de marquage épistémique - comme hyperonyme [...]. » Dendale et Tasmowski (1994: 4).

(11) « Toute opération (intellectuelle) par laquelle on admet une proposition dont la vérité n'est pas connue directement mais en liaison avec d'autres propositions déjà tenues pour vraies. " (Lalande, sv. inférence).

(12) "Décision mentale par laquelle le contenu d'une assertion est posé à titre de vérité " (NPRE, sv. jugement, $4^{\circ}$ ) 
(7) Jules étudie.

qui s'interprète comme décrivant un état de choses.

Les enchainements (5) et (6) montrent que l'emploi de censé ne relève pas, en ce qui concerne la prédication opérée par l'infinitif, d'une logique de la description des états de choses mais de celle de la description d'une possibilité de principe, qui se vérifie ou non ( $c f .(6))$ et sur l'existence empirique de laquelle il est loisible de s'interroger ( $c f$. (5)) : énoncer $X$ est censé INF ne garantit pas 1'actualisation $X I N F$. L'enchainement dans (6) montre plus spécifiquement que censé ne dit rien du jugement du locuteur en termes de vérité concernant $X I N F$.

Ce qui explique que censé s'associe à une contre-vérité / réalité :

(8) Désolé..., on est lundi et le lundi, je suis censé être ailleurs. Revenez demain.

Si donc il est un domaine où peut s'évaluer la vérité de $p$, c'est celui des principes, et donc des possibles, étranger à toute actualisation.

Deux conséquences découlent de cette signification de censé. D'une part, censé peut introduire dans l'énoncé une indication médiative "négative ": censé signale en creux que $X I N F$ n'est pas une information obtenue par l'expérience (d'un état de choses) ; mais, en soi, censé ne dit rien du mode d'accès à $X I N F$. D'autre part, en situant $X I N F$ dans le domaine des principes et du possible, censépeut participer au marquage de l'attitude épistémique du locuteur ${ }^{(13)}$.

\subsubsection{Valeurs de la signification censé1}

Censé signifie l'existence d'une conception à propos du référent du contrôleur, $X$ : celui-ci est conçu comme doté des propriétés (procès ou qualités) que dénote $I N F$. Les énoncés se paraphrasent bien par On attend d'un X que INF.

On distingue deux cas.

\section{Cas 1. Les énoncés généraux}

(9) Un étudiant est censé étudier.

(10) Le décideur rationnel est censé savoir ce qu'il veut. (Google)

(9)-10) acceptent une interrogation portant sur censé:

(11) Un étudiant est-il censé étudier?

(12) Le décideur rationnel est-il censé savoir ce qu'il veut?

et ce contrairement, par exemple, à devoir épistémique :

(13) Maintenant, Pierre doit être arrivé. / *Maintenant, Pierre doit-il être arrivé ?

Ceci indique que censé, intra-prédicatif comme devoir en (13), fait partie du rhème dans (9)-(10).

Le contenu $X I N F$ ne se donne pas comme une création originale du locuteur ; $X I N F$ est préconstruit. En (9), le prédicat étudier est construit sur une relation d'inhérence à la signification stéréotypique du lexème étudiant ${ }^{(14)}$; en (10), savoir ce qu'il veut est un stéréotype de décideur (par définition pourrait " préfixer» (9)-(10)). C'est en ce sens que le contenu de la prédication censé résulte d'une inférence.

(13) $C f$. note 9.

(14) Stéréotypique en ce sens qu'un étudiant qui n'étudie pas n'en est pas moins étudiant. 
La relation aux stéréotypes fait qu'en (9)-(10) la transformation négative produit un discours peu cohérent :

(14) 'Un étudiant n'est pas censé étudier / Le décideur rationnel n'est pas censé savoir ce qu'il veut.

Le jugement est identique pour (15) qui ne répond pas au stéréotype :

(15) ? Un médicament est censé détériorer la santé.

Par l'intermédiaire des stéréotypes qu'elle convoque dans ces énoncés, l'unité censé, présente le contenu de $I N F$ comme attribuable à un énonciateur ${ }^{(15)}$.

Comparées aux versions dépourvues de être censé ${ }^{(16)}$ :

(16) Un étudiant, ça étudie.

(17) Le décideur rationnel sait ce qu'il veut.

(9)-(10) font, d'une part, explicitement allusion au fait que INF est conceptuellement une propriété nécessaire de $\mathrm{X}$; censé fait donc allusion au mode d'existence de ce que dénote INF : c'est une propriété de X théoriquement, idéellement, associée à étudiant, en tant qu'étudiant désigne un rôle.

(9)-(10) - et cela est lié - font d'autre part explicitement allusion à la possibilité de cas particuliers ne vérifiant pas le prédicat étudier. (D'où l'effet de sens de non-prise en charge qui peut se surajouter aux énoncés, effet fréquent hors d'un contexte qui permette d'accéder exactement à ce que veut dire le locuteur. Ainsi (9) a une valeur d'aphorisme. Selon les discours, l'énonciation de (9) pourra correspondre à la mise en place de l'aphorisme comme prémisse à un discours, ou au rappel de l'aphorisme ; dans ce second cas, l'énonciation de (9), par exemple face à de nouveaux étudiants, s'associera à une attente (sinon à une injonction) du locuteur; en revanche, face à un étudiant peu enclin au travail, elle signifiera l'exception au principe.

Au final, censé 1 renvoie régulièrement à un énonciateur, cela ne signifiant pas, on l'a vu, que le locuteur lui « emprunte »XINF.

\section{Cas 2. Les énoncés spécifiques}

\section{a) Le référent du contrôleur est un humain ; il est vu sous la facette rôle}

(18) Il fait des paysages, des personnages, des animaux. S'il voulait, il pourrait exposer.

- Oui, oui. Et vous apprenez le métier?

- Oui.

- Ou vous êtes censé l'apprendre. Parce que si vous passez vos après-midi aux courses...

- Le matin, je broie des couleurs. Je nettoie les brosses. (ROMAINS, J., Les Hommes de bonne volonté, 1932)

Censé est rhématique ; ceci découle de l'opération de reformulation : quand censé est énoncé, la prédication $X$ apprendre le métier fait partie du thème ; pour preuve : la suppression de censé est impossible ( 'Ou vous l'apprenez).

La prédication de censé corrige l'assertionj'apprends le métier et oppose deux

(15) Le terme correspond à la définition $i$ que défend Anscombre (2006:354) et non à la $i i$ qu'il rejette : «i.) Les énonciateurs ne font pas d'actes. En fait, ils ne font rien du tout, ils représentent des points de vue. [...] ;ii) Les énonciateurs ne sont pas des points de vue, mais sont bel et bien les auteurs de phrases. "

(16) (9) nécessite une reprise en ça pour être naturel. 
univers de croyance : celui de l'interlocuteur où la proposition apprendre le métier est vraie, et celui du locuteur où elle est présentée comme discutable.

L'énonciation de censé s' autorise d'une inférence qui mobilise une information détenue par le locuteur (vous passez vos après-midi aux courses...) et un stéréotype (il affleure dans le passage introduit par parce que...) selon lequel apprendre le / un métier et s'adonner à des loisirs sont incompatibles, ce stéréotype étant associable à un $O N$-énonciateur ${ }^{(17)}$.

Une paraphrase approximative du segment contenant censé serait : on a dans l'idée que vous l'apprenez. Censé construit l'idée que le référent de vous ne peut, d'un certain point de vue, être conçu autrement que comme « apprenant le métier », cela en vertu de son rôle d'apprenti, qui lie nécessairement apprenti et apprendre le métier. Le on en question ici est un $O N$-Enonciateur indéterminé.

En (18), le locuteur peut faire partie du $O N$-énonciateur qui admet le stéréotype ; il ne fait pas partie du $O N$-énonciateur qui admet que ce stéréotype est applicable à Vous, vu sous la facette « apprenti».

\section{b) Le référent du contrôleur est un objet vu sous l'angle de sa fonction}

(19) Les tringles en fer rouillé qui sont censées protéger les tableaux du Louvre contre les attentats de cette nature sont une défense bien illusoire et d'ailleurs trop peu systématique [...] (REAU, L, Archives, 1909)

Censé se glose par prévu, conçu pour (parfois, destiné à). Ces gloses verbalisent, d'une part, une visée, d'autre part, l'attribution anticipée d'une fonction à un objet.

La suppression de censé n'est pas impossible au sens où elle l'est pour (18),

(20) Les tringles en fer rouillé qui protègent les tableaux du Louvre contre les attentats de cette nature sont une défense bien illusoire.

mais elle gomme l'idée de visée: 1'expression définie Les tringles en fer rouillé semble alors référer strictement aux objets empiriques qu'elles désignent, non plus à la facette fonction dévolue aux objets qu'elle a dans 1'original et qui est donc introduite par censé.

Dans (19), c'est, a minima, de la présence même des tringles que le locuteur conclut à la fonction protectrice qui leur est dévolue (suivant un « raisonnement » du type : s'il y a des tringles devant ces tableaux-là, c'est pour les protéger); mais c'est dans un autre univers (celui des concepteurs de l'installation de tringles devant les tableaux, concepteurs dont on ignore si le locuteur a fait partie ${ }^{(18)}$ ) que cette relation entre les tringles et la protection de ces tableaux précis a été établie sur le mode d'une visée liée à la fonction de tels objets. (19) met en relation l'univers des concepteurs dans lequel la proposition Des tringles en fer protègent les tableaux est une proposition générale possiblement vraie que les faits doivent valider et celui du locuteur où la proposition spécifique et référée à un monde actuel est plus ou moins vraie (cf. une défense bien illusoire).

La présence de censé en (18)-(19) fait de XINF une proposition non actualisée en ce sens qu'elle est dotée d'une valeur en dehors d'une confrontation au réel et antérieurement à cette confrontation.

(17) Nous adoptons une suggestion d'Anscombre (2006:360) : « [...] il faudrait en fait distinguer des $O N$-énonciateurs - auteurs d'énonciations virtuelles, et des $O N$-locuteurs, auteurs d'énonciations présentées comme effectives. "

(18) D'où le a minima utilisé plus haut. 


\subsection{Censé2}

Censé signifie unjugement du locuteur, précisément, sa décision de doter, arbitrairement, au moment de l'énonciation, la proposition $p(X I N F)$ du statut de vérité, et donc d'attribuer à $X$ le prédicat $I N F$. L'attribution de la valeur « vrai » à $p$ est indépendante d'une validation par le réel.

Censé - qui dénote alors une action du locuteur - permet l'établissement de principes, locaux ou généraux ; l'énoncé se glose par : Il est décidé que / Je décide que ; censé accepte une substitution par considéré comme ${ }^{(19)}$.

Cette signification apparait dans deux contextes, contexte à référent $X$ spécifique (21), contexte à référent $X$ intensionnel (22) :

(21) «Considérons les énoncés suivants où les expressions en gras sont censées être coréférentielles :

Emile pense que Sophie l'aime.

Emile espère que Sophie l'aimera un jour. » (RUWET, N., "En et y...», Langue Française, n 97, $1990: 51$ )

(22) L'héritier qui renonce est censé n'avoir jamais été héritier. [...] (Article 805, Code civil)

$X$ être censé INF se paraphrase, dans (21), plutôt par nous considérerons que $X$ être INF, dans (22) par nous posons que X doit désormais être considéré comme.

(21) illustre une convention locale, créatrice d'un cadre de validité pour l'interprétation de la suite du discours ; (22) illustre une convention générale, une loi, créatrice d'un cadre pour des situations éventuelles et futures. Censé souligne la dimension décisionnelle, quasi performative de l'énonciation (d'où, sans doute, que être censé accepte le futur: Considérons les énoncés suivants où les expressions en gras seront censées être coréférentielles, L'héritier qui renonce sera censé n'avoir jamais été héritier) ; du performatif, (21)-(22) présentent une caractéristique pragmatique essentielle, les conditions sur le sujet parlant (être du monde) : le rédacteur d'article dans (21) jouit du pouvoir de fixer certaines interprétations ; le Législateur dans (22), être extra-linguistique fantasmé, du pouvoir de légiférer.

Supprimer être censé INF est impossible en (21) (Considérons les énoncés suivants où les expressions en gras sont coréférentielles) : cela entraîne la fausseté de la proposition et modifie la nature de l'acte effectué (énonciation d'un jugement en (21), l'énoncé devient énonciation d'une évaluation du statut référentiel des « expressions soulignées »). Censé souligne la conventionalité du choix opéré entre les possibles et renvoie automatiquement à un autre point de vue sur les « expressions soulignées ».

Délicate pour (22) mais non impossible (L'héritier qui renonce n'a jamais été héritier pourrait encore être lu comme une loi), la suppression fait cependant mesurer l'apport de censé qui est encore de signifier la dimension conventionnelle de $X I N F^{(20)}$, c'est-à-dire d'en signifier le mode d'existence : XINF est une proposition dont l'assertion n'est pas validée par le réel. On observe aussi que censé conduit à interpréter $n$ 'avoir jamais été héritier non comme une qualification mais comme une requalification de $X$, ce qui atténue l'incohérence d'une assertion

(19) Censé, ée 1. «Considéré comme» en vertu d'une fiction de la loi. Ex. C. civ., a. 883. Comp. réputé, assimilation. (Cornu dir. 2000).

(20) (22) parait, au profane, énoncer une contre-vérité (qui renonce à être héritier a bien étéhéritier ; $c f$. le « présupposé » de renoncer). 
(comment peut-on être dit n'avoir jamais été ce que l'on a été ?), contraire au sens commun d'un $O N$-énonciateur.

\subsection{La part du contexte dans l'interprétation d'un énoncé comportant censé : effets de sens}

Nous avons distingué deux significations de censé INF, mais il n'est pas toujours simple d'associer tel énoncé à l'une ou l'autre de ces significations. Voyons cela.

- Un des problèmes est de savoir ce que signifie l'énoncé contenant censé. Un exemple :

(23) Ces analyses sont censées montrer les vertus descriptives de notre système, de même que certains de ses vices cachés.

Il y est question d'évaluer un système proposé par le locuteur. Mais ce locuteur veut-il dire qu'il propose lui-même un moyen d'évaluation (censé2) ou que ce moyen a été proposé par un tiers (censé1) ? Dans son contexte, (23) n'est plus ambigu :

(24) Le premier objectif devrait être réalisé dans le chapitre 2, où sera présenté notre système descriptif. Le second objectif devra être réalisé par les trois analyses concrètes de marqueurs épistémiques que nous présenterons dans la seconde partie. Ces analyses sont censées montrer les vertus descriptives de notre système, de même que certains de ses vices cachés. (Dendale, P., Le marquage épistémique de l'énoncé,1991: 32)

Il exclut l'interprétation « évaluation par autrui ».

- Cet exemple permet deux observations. La première interprétation de (24) s'associe à l'idée que le locuteur estime vraie la proposition ces analyses-montrer (elles ont pour lui la capacité d'évaluer); dans la seconde, on interprète plutôt que le locuteur leur dénie cette capacité. Ceci indique que censé n'est pas intrinsèquement associé à l'évaluation épistémique.

L'exemple montre aussi que censé peut être associé à un trait médiatif d'emprunt (nous entendons par là, non tout ce qui ressortit à la polyphonie, mais l'utilisation plus ou moins libre d'un discours effectif ${ }^{(21)}$ ) ; en l'occurrence il y a emprunt au discours d'un locuteur identifiable ${ }^{(22)}$.

Cela est différent de l'interprétation associée à (25) qui décrit un comportement social « ritualisé »

(25) ... de même qu'un grand raout dans le faubourg Saint-Germain n'est plus un raout s'il y a une comédie ou de la musique. Il faut qu'on soit censé venir simplement - y eût-il cinq cents personnes - faire une visite à la princesse de Guermantes, par exemple. (Proust, M., À la recherche du temps perdu. Du côté de Guermantes 2, 1921)

L'idée de simulacre ${ }^{(23)}$, donc de faux, est introduite en dehors de censé, par la co-présence de venir faire simplement une visite et de cinq cents personnes, nom-

(21) Voir Anscombre (2006:360).

(22) Que censé INF puisse s'associer à un marqueur d'emprunt (au sens large), tel Il parait que, sans qu'il y ait redondance montre que censé INF n'est pas spécifiquement associé à un type particulier de marquage évidentiel. Ex. : J'espère que ça ne va pas durer 2 mois comme ça et qu'il va changer de position. Je sais qu'il s'est déjà retourné, le médecin me l'a confirmé dernièrement et je le sens sur mon côté droit. Il parait qu'il est censé se placer sur le côté gauche. On verra bien (n9ufmois.blogspot.com/2008_05_01_archive.html).

(23) Censé est également associé aux simulacres dans des textes d'anthropologie et dans certaines didascalies: Au Queensland, les fillettes se mettent sur la nuque un bâton fourchu qui 
bre incohérent avec l'idée de simple visite. Censé ici encore marque que INF (et ses compléments : venir simplement en visite) est sans réalité empirique et n'existe que sur le mode du faire comme si, de l'apparence. INF et l'obligation qui lui est liée ( $c f$. il faut) repose sur une convention sociale dont on peut douter ici qu'elle ait été explicitement verbalisée mais qui est sans doute le contenu (propositionnel) d'un désir de la communauté qui s'y plie, d'un $O N$-énonciateur donc.

- L'observation des exemples nous conduit à conclure que l'essentiel de la signification de censé semble tenir dans les deux traits suivants : présenter $X I N F$ comme non actuel et évoquer un énonciateur, énonciateur qui n'est pas nécessairement la source évidentielle de XINF. Nous rangeons donc censé dans la modalité, non que censé dise systématiquement quelque chose de l'attitude du locuteur vis-à-vis de la vérité de $X I N F$, mais parce que censé dit quelque chose de la nature de $X I N F$ : c'est une proposition non actuelle (la non-actualité d'une proposition n'impliquant pas sa fausseté). Bref, censé est un modalisateur intra-prédicatif dont le rôle est de qualifier le mode d'existence de la proposition à laquelle il s'associe, de la catégoriser ${ }^{(24)}$.

\section{RÉPUTÉ}

Nous distinguerons deux significations, selon l'agent sémantique de réputé : soit cet agent est un ensemble d'individus formant une communauté, et dans ce cas réputé présente $X I N F$ comme une croyance (réputé1) ; soit l'agent est le législateur et réputé correspond à un jugement, (réputé2). Seul réputé2 correspond sémantiquement au verbe réputer ${ }^{(25)}$.

\section{Réputé1}

a) Dans l'énoncé $X$ (être) réputé INF, l'unité réputé présente le contenu propositionnel $p$ (construit par $X I N F$ ) comme emprunté.

Réputé 1 ne relève cependant pas du discours rapporté : 1'unité signale que celui qui énonce $X$ (est) réputé INF a acquis $p$ par le biais d'un dire, mais elle ne permet en rien de représenter un acte d'énonciation. Réputé 1 pourrait en revanche être assimilé à un marqueur de modalisation en discours second (Authier-Revuz 1992 : 39) : dans certains exemples du corpus Frantext, réputé apparait comme un commentaire du locuteur sur l'énoncé ou une partie de l'énoncé, signalant 1'emprunt de $X I N F$ à un autre discours (ce commentaire étant syntaxiquement intégré) :

(26) 1000 essais furent accomplis avec des sujets réputés ou prétendant posséder des facultés parapsychiques particulières. (AMADOU, R., La Parapsychologie, 1954)

Mais dans d'autres cas, réputé1 fait partie de la prédication ${ }^{(26)}$ : réputé est alors

est censé représenter un bébé gigotant sur l'épaule de sa mère. (LOWIE R.-H, Anthropologie culturelle, 1936)

(24) Notons que être censé INF est rangé dans la modalité par Gaatone (1998:81); pour une position opposée, voir Borillo (2005).

(25) D'une part, réputer peut avoir pour sujet un SN référant à des êtres individualisés (par ex. :

C'était sa luciférienne vengeance de la destruction du couple passionné, magnifique, qu'ils formaient, Angiolina et lui, et que, l'un et l'autre, ils réputaient indestructible. MATZnefF, G., Ivre du vin perdu, 1981), alors que réputé 1 a un agent référant à une collectivité ; d'autre part, réputer suppose un jugement (voir note 12), tandis que réputé correspond à une croyance concernant un caractère singulier du référent de $X$.

(26) X être réputé INF est glosable par « $\mathrm{X}$ a la réputation de INF »; réputation partage avec ré- 
susceptible de recevoir les mêmes complémentations qu'un participe intégré à la prédication (compléments adverbiaux : réputé en ce temps, réputé par une association, réputé selon un symbolisme...) et réputé INF peut être pronominalisé en le dans une comparative :

(27) L'arrière-grand-père, le grand-père et le père de Paul sont réputés, comme il l'est lui-même, avoir été ou être des personnages " regardants», sinon avares... (YOURCENAR, M., Le Labyrinthe $d u$ monde, 1988 ; nous soulignons.)

Au total, réputé1 correspond toujours à un emprunt mais peut avoir deux statuts : soit il modalise l'énoncé (le locuteur indiquant ainsi que $X I N F$ est emprunté), soit il prédique.

b) Le dire auquel renvoie réputé est d'un type particulier : il exprime une croyance (collective) en la vérité de $p$; cette croyance concerne un caractère qui distingue $X$ : réputé présente en effet $I N F$ comme un procès qui constitue un caractère distinctif de $X$; c'est net lorsque le procès est à même de susciter (dans un système de valeur / pensée donné) des jugements positifs ou négatifs sur $X$ - par exemple, guérir telle maladie, posséder des facultés particulières ( $c f$. supra, (26)) mais $I N F$ en lui-même peut ne pas être axiologique et correspondre par exemple à une évaluation quantitative ; le caractère distinctif de $I N F$ apparait alors grâce au contexte - en (28), le fait que les indentations de la queue soient « au nombre de 6 ou de 7 selon les cas » est ce qui met l'animal en relation avec la voute céleste :

(28) Un animal dépourvu d'utilité pratique est souvent invoqué dans les rites : la tortue à queue en dents de scie. Son importance serait à jamais inintelligible, si l'on ne savait pas par ailleurs que le chiffre 13 possède pour les Osages une valeur mystique. Le soleil levant répand 13 rayons, qui se répartissent en un groupe de 6 et un groupe de $7[\ldots]$. Or, les indentations de la queue de cette espèce de tortue sont réputées être au nombre de 6 ou de 7 selon les cas, la poitrine de l'animal représente donc la voûte céleste, et la ligne grise qui la traverse, la voie Lactée. (LÉviStrauss, C., La Pensée sauvage, 1962)

Ce caractère distinctif est nécessairement acquis avant le moment où (est) réputé est valide : il ne peut pas survenir postérieurement à la réputation ${ }^{(27)}$.

c) Cette croyance concernant le référent de $X$ est assignée à une collectivité qui peut ne pas être identifiable; c'est le cas en (29) où rien ne permet de savoir si la croyance en question est circonscrite à l'espace spatio-temporel délimité par le cotexte (dont le temps verbal) ou non, autrement dit si $p$ est valide seulement pour un ensemble d'individus vivant à l'époque évoquée ou si $p$ l'est encore pour certains individus au moment de l'énonciation :

(29) Pour le liquide, de nombreuses boutiques, caves de ceci, caves de cela, débitaient, à la tireuse ou au tonneau, l'aramon ou le blanc liquoreux, réputé donner à l'haleine des buveurs endurcis une senteur de gaz acétylène. (SIMONIN, A., Confessions d'un enfant de la Chapelle, 1977)

Le contexte peut fournir des éléments spécifiant l'énonciateur de cette opinion, par le biais, par exemple, d'un SNP de temps et / ou de lieu en tête de proposition, définissant un « univers de discours » (Martin 1987:38-39) : la vérité de l'énoncé

puté les traits « dire collectif exprimant une croyance » et « évaluation » (cf. par ex. «Opinion favorable ou défavorable attachée à quelqu'un ou à quelque chose. », TLFi, A).

(27) Dans : X est réputé pouvoir + infinitif, seul le procès signifié par l'infinitif est postérieur ; la capacité (signifiée par pouvoir) ne l'est pas. 
$X$ être réputé INF est valide dans cet univers, qui, par métonymie, permet de définir l'image d'univers ( $c f$. Martin, $1987: 19-21$ ) où $p$ est vrai (image d'univers d'un ensemble d'énonciateurs caractérisés par le fait qu'ils vivent dans le Hoggar à l'époque de Denys) :

(30) Denys m'apprenait donc que [...] et que, dans ce Hoggar, du moins à son époque, la noble dynastie neptunienne était réputée se perpétuer encore. (BENOIT, P., L'Atlantide, 1919)

d) Le point de départ de la réputation, sa cause, peut être explicité par un complément prépositionnel, en par ou en selon :

(31) A l'origine, il [saint Erasme] est patron des marins ; l'image le marquera par un attribut : le treuil avec son câble enroulé. Mais le sens connu se perd. Reste l'image : il faut lui en trouver un autre; elle le suscite elle-même. Que peut être ce bizarre instrument sinon celui de son supplice, qui a fait de lui un martyr? Puisque treuil il y a, on supposera et bientôt on affirmera que les tortionnaires s'en sont servis pour dévider ses intestins. Encore un pas, et saint Erasme sera réputé guérir les coliques, par une association spontanée. Ainsi se fonde, sinon un culte, du moins une dévotion. (HuYGHE, R., Dialogue avec le visible, 1955 ; nous soulignons.)

Au total, ce qui distingue réputé1 de censél, outre le type d'évidence (emprunt pour l'un, inférence pour l'autre), c'est :

- le fait que, avec réputé1, INF correspond à une évaluation, positive ou négative, ce qui n'est pas nécessairement le cas avec censé ;

- la contrainte concernant la situation dans le temps du procès dénoté par l'infinitif par rapport au moment où (être) réputé est valide : ce procès est toujours antérieur ou simultané (et peut se prolonger ultérieurement).

\subsection{Réputé 2}

Dans notre corpus cette signification est attestée dans des textes à caractère juridique, mais elle peut l'être ailleurs, notamment pour fixer ou rappeler des positions théoriques. Dans la terminologie juridique, réputé a les acceptions suivantes (cf. de Cornu, dir.) :

1. "Présumé par la loi »Ex. Tout bien est réputé acquêt de communauté (C. civ., a. 1402). Comp. censé. V. présomption.

2. Considéré par la loi comme. V.fiction. Comp. assimilation.

La loi est définie comme exprimant la volonté d'une communauté (cf. définition de loi dans Cornu, dir.) ; réputé2 renverrait au Législateur, seul habilité à légiférer, mais qui enregistre la volonté d'une communauté.

Deux cas sont à distinguer.

\subsubsection{Réputé 2a}

Réputé renvoie à une décision antérieure ; en (32), c'est une loi du 12 juillet 1927 qui a posé que tel était le cas - indépendamment de toute " réalité » :

(32) Une loi du 12 juillet 1927 lui impartissait pour faire son choix un certain délai, passé lequel il était réputé avoir opté pour le mandat le plus récent et avoir démissionné du mandat le plus ancien. (VEDEL, G., Manuel élémentaire de droit constitutionnel, 1949) 


\subsubsection{Réputé $\mathbf{2 b}$}

Il s'agit de l'emploi de réputé dans des énoncés qui fixent ce qui sera désormais tenu pour vrai - et en ce sens, on a affaire à des énoncés performatifs ; réputé $2 \mathrm{~b}$ parait proche de censé2 (cf. Cornu, sv. Réputé, qui renvoie à censé, et dans l'article fiction : «artifice de technique juridique, mécanisme souvent annoncé par les expressions « est réputé...» « censé ». [...]»). Les cotextes sont identiques à ceux qui ont été décrits pour censé 2 (voir supra) :

(33) ...pour la détermination de l'époque à partir de laquelle ils [les engagés] servent au delà de la durée légale, les engagés sont réputés avoir les mêmes obligations légales d'activité que celles imposées aux hommes de cette fraction de classe. (Loi relative au recrutement de l'armée, 1928)

Du point de vue syntaxique, réputé $2 \mathrm{~b}$ présente les caractéristiques suivantes :

- réputé n'est pas en fonction d'épithète liée ; s'il est employé dans un texte de loi avec cette fonction, il renvoie à une décision fixée ailleurs ${ }^{(28)}$ (il s'agit donc du type réputé $2 \mathrm{a})$;

- être est au présent ou au futur - dans un système hypothétique ou non ;

- être réputé est le verbe d'une proposition formant un énoncé à part entière (proposition non régie, relative explicative).

On peut envisager d'établir un lien entre réputél et réputé2, si l'on considère la loi comme l'ensemble des discours émanant de la volonté d'une communauté. Réputé 2 , en ce sens, relèverait de l'emprunt, de même que réputé1, mais au sens large du terme : réputé 2 a renvoie au discours que constitue un texte déjà existant ; réputé $2 \mathrm{~b}$, en tant que discours qui décrète, énonce performativement la loi et donne - contextuellement - le contenu de cette loi comme la « représentation » de la volonté d'un $O N$-énonciateur (antérieure au texte de loi où figure l'occurrence de réputé). L'interprétation des occurrences de réputé2 reste cependant malaisée : elles relèvent d'un usage spécialisé (juridique) dont nous n'avons pas la performance.

\section{SUPPOSÉ}

On propose de distinguer trois significations : dans la première, supposé1, on retrouve un trait présent dans les emplois réputé2 et censé2 : le trait/décision/; la seconde (supposé2) comporte, comme censé1, le trait/inférence/; la dernière (supposé3) ne comporte pas de trait médiatif: elle n'indique pas la façon dont $p$ a été acquis.

\subsection{Supposé1}

La proposition $p$ que constitue $X I N F$ est présentée comme le résultat d'une décision prise, indépendamment de toute évaluation de la vérité de $p$. Elle constitue une hypothèse : il est admis, au moins provisoirement, que tel est le cas, éventuellement dans un objectif précis ( $c f$. en (34): «pour simplifier»). Ce type se rencontre notamment dans les textes théoriques, où la décision s'inscrit dans un raisonnement (elle en marque une étape) :

(34) Supposons au contraire que, le frottement étant négligeable, notre masse fluide

(28) Avec une autre construction que celle qu'on étudie ici : [...] Dans tous les cas, en ce qui concerne les biens réputés indivis en application de l'article 1872 (alinéas 2 et 3)... (Code civil - Art. 1872-1) 
soit le siège de brassages intérieurs, ces brassages étant supposés conserver, pour simplifier, la symétrie de révolution de notre masse autour de... (POINCARÉ, H., Leçons sur les hypothèses cosmogoniques, 1911)

mais ce peut être aussi une base arbitrairement choisie pour une action et qui conditionne la façon dont cette action est réalisée :

(35) Pour ce genre de chronique, le problème aujourd'hui se pose tout différemment. La lectrice est supposée tout ignorer de la cuisine ; par contre, elle peut suivre des indications très précises, grâce à la balance, aux paquets ou portions standard fournis par les marchands... (La Civilisation écrite, dir. J. Cain, 1939)

X être supposé INF apparait comme le passif de supposer XINF au sens de «poser, considérer quelque chose comme vrai ».

A ce type s'apparente $X$ (être) supposé INF en didascalies ; par convention, on admet que $X$ a la propriété dénotée par $I N F$ :

(36) SIR HAROLD, au cheval supposé être dans la coulisse : Tranquille, Bijou ! (À Saïd.) Tu as besoin de faire des économies? (GENET, J., Les Paravents, 1961)

Dans des emplois tels que (34)-(35), la substitution de censé à supposé entraine un changement sémantique net : il ne s'agit plus alors d'une hypothèse, mais d'une propriété attendue de $X$; la différence est plus ténue, hors contexte, pour (36).

\subsection{Supposé2}

(Etre) supposé XINF signifie le résultat d'une opération mentale consistant en une inférence faite à partir d'un indice ; en (37) l'indice sur lequel se construit l'inférence, faite par des juges, est explicité les draps:

(37) C'est l'un d'eux qui a pu, par exemple, venir dans le chemin de ronde détacher les draps, pendant dans la rue, grâce auxquels je suis supposé avoir franchi le grand mur. Mais cette hypothèse rocambolesque (car je n'avais aucun moyen d'établir un contact quelconque avec les hospitalisés non prisonniers...) (MENDĖS-FRANCE, P., Euvres complètes. 1922-1943/1984)

On est proche ici d'un rapport de pensée ou de discours ; $p$ est vrai (ou probablement vrai) pour l'agent de l'inférence, mais, ici, faux pour le locuteur ( $c f$. «cette hypothèse rocambolesque »).

$X I N F$ peut aussi être tiré de propriétés inhérentes à $X$ ou qui lui sont attachées conventionnellement :

(38) Au fait, dis-je, très Alexandre Dumas, comment m'as-tu retrouvé ?

- Ton ancienne concierge. Elle était supposée faire suivre ton courrier, non ?

«Supposée » est bien le mot. A moins que personne ne m'ait écrit.

- En effet..., dis-je.

(Benoziglio, J.-L., Cabinet portrait, 1980)

La proposition $p$ «ton ancienne concierge fait suivre ton courrier » est tirée du stéréotype attaché à la fonction que le nom concierge désigne : faire suivre le courrier est un acte attendu d'une concierge ; la reprise (" "supposée" est bien le mot») signale que $p$ est sans correspondant dans le monde : $p$ est de l'ordre de l'attente.

Dans ces exemples, supposé2 oppose l'univers de croyance de l'énonciateur de supposé à l'univers de croyance du locuteur : en (37), la proposition $p$ («j'ai franchi le grand mur ») est vraie ou probablement vraie dans l'univers de croyance des juges, fausse dans celui du locuteur ; en (38), la proposition générale « une con- 
cierge fait suivre le courrier » est vraie pour un $O N$-énonciateur, la proposition spécifique « ma concierge a fait suivre mon courrier», fausse pour le locuteur. Cette opposition n'est cependant pas nécessairement attachée à supposé2, la valeur de vérité de $p$ pouvant restée indéterminée dans l'univers du locuteur.

\subsection{Supposé3}

La proposition correspondant à $X I N F$ est vraie dans une image d'univers, mais le locuteur ne prend pas parti sur la vérité de la proposition; il en résulte un effet d'atténuation : le locuteur ne prend pas $p$ en charge.

L'énonciateur de $p$ peut être un $O N$-énonciateur :

(39) Quand avait-elle été heureuse ? Où étaient ces moments de bonheur dont l'enfance est supposée être faite, où étaient ses vacances? (GREEN, J., Adrienne Mesurat, 1927)

il peut aussi être reconstituable à partir d'éléments contextuels, à savoir dans (39) le sujet du verbe principal (beaucoup de tribus d'esquimaux) :

(40) Beaucoup de tribus d'esquimaux considèrent criminel de manger du phoque et du caribou à un même repas, l'infraction du tabou étant supposée menacer les réserves alimentaires (voir plus haut). (LowIE, R-H., Anthropologie culturelle, 1936)

On a ici un des cas rares où réputé serait substituable à supposé; cette possibilité tient au fait qu'il est question ici d'une croyance collective, selon laquelle $X$ a telle propriété. La commutation atténuerait cependant 1'effet de non prise en charge que l'on a avec supposé.

\section{Conclusion}

Les dictionnaires consultés incitent à penser que les unités sous étude sont synonymes. Nos hypothèses de départ étaient, classiquement, que cette synonymie n'est pas totale et que les différences sont à chercher essentiellement du côté de la question de la « source de l'information".

Au final, il importe de noter que même en restreignant l'étude à une seule structure, les unités sont polysémiques; dans cet article, première étape de notre travail, nous avons choisi d'envisager la synonymie non entre les unités elles-mêmes, mais entre les diverses significations identifiées pour chaque unité.

Ainsi, les trois unités ont-elles des emplois qui relèvent de la décision : censé2, réputé 2 et supposé1 (elles peuvent alors relever d'un vocabulaire «spécialisé »). Cela dit, il n'y a de proximité sémantique qu'entre censé 2 et réputé2 (au moins dans les textes de lois, ces deux unités signifient une intervention sur le réel); quant à supposé1, il n'appartient pas à la terminologie juridique, et ses emplois ne correspondent pas à une décision modifiant le réel.

Par ailleurs, supposé2 et censé1, sont très proches sémantiquement : ils supposent, l'un et l'autre, une opération d'inférence.

Restent, en revanche, totalement isolés, réputé 1 qui se singularise par le trait médiatif/emprunt/ au sens strict, et supposé3, en ce qu'il signale la position du locuteur vis-à-vis de $X I N F$, sans comporter de trait médiatif (au sens strict) ${ }^{(29)}$.

(29) Notre travail n'a pu, hélas, profiter de celui de J. François (2003) sur censé : nous en ignorions 1'existence. 


\section{Références bibliographiques}

ANSCOMBRE, J.-C. (2006) : «Stéréotypes, gnomicité et polyphonie : la voix de son maitre », in Perrin, L. dir., Le sens et ses voix, dialogisme et polyphonie en langue et en discours, Recherches $\mathrm{n}^{\circ} 28$, Metz, 349-378.

AUTHIER-REVUZ, J. (1992-1993) : "Repères dans le champ du discours rapporté », L'Information grammaticale $55: 38-42$, et $56: 10-15$.

Borillo, A. (2005) : «Peut-on identifier et caractériser les formes lexicales de l'aspect en français? », in : Bat-Zeev Shyldkrot, H. et Le Querler, N. dir. Les périphrases verbales, Linguisticae investigationes, supplementa 25, 83-102.

Dendale, P. \& TAsmowski, L. (1994) : «Présentation », Langue française, Les sources du savoir, $\mathrm{n}^{\circ} 102,3-7$.

FRANÇOIS, J. (2003) : La prédication verbale et les cadres prédicatifs, Louvain / Paris, Peeters.

GAATONE, D. (1998) : Le passif en français, Bruxelles : Duculot.

Goosse, A. \& Grevisse, M. (2007) : Le Bon Usage, $14^{\mathrm{e}}$ éd., Bruxelles / Louvainla-neuve : De Boeck / Duculot.

Gross, G. (1975) : Méthodes en syntaxe, Paris : Hermann.

LE GOFFIC, P. (1993) : Grammaire de la phrase française, Paris : Hachette.

MARTIN, R. (1987) : Langage et croyance, Bruxelles / Liège : P. Mardaga.

Wilmet, M. (2003) : Grammaire critique du français, Paris : Hachette.

\section{Dictionnaires de langue}

Ac. 9 : Dictionnaire de l'Académie française, $9^{e}$ éd., http://atilf.atilf.fr/academie9.htm

GLLF : Grand Larousse de la langue française. Paris : Larousse.

GR : Le Grand Robert de la langue française, Dictionnaires Le Robert, 2001.

Lexis: Larousse de la langue française, Larousse, 1989.

NPRE : Nouveau Petit Robert électronique 2007, Dictionnaires Le Robert / Sejer, 2006.

TLFi : Trésor de la langue française informatisé, Atilf-CNRS : http://atilf.atilf.fr/ tlf.htm

\section{Dictionnaires spécialisés}

CORNU, de, G. dir. (2000) : Vocabulaire juridique, $8^{\mathrm{e}}$ éd. rev. et augm., PUF [ $1^{\mathrm{re}}$ éd. 1987].

LALANDE, A. (1991) : Vocabulaire technique et critique de la philosophie, Paris : P.U.F., Quadrige [ $1^{\text {re }}$ éd. 1926]. 